\title{
Potential Active Ingredients of Traditional Chinese Medicine NaoMaiTong for Ischemia Stroke Treatment
}

Yu Zhang ${ }^{1}$, Xu-lei Fan ${ }^{1}$, Li Lu ${ }^{1}$, Shu-mei Wang ${ }^{1,2,3 *}$

${ }^{1}$ Department of Traditional Chinese Medicine, Guangdong Pharmaceutical University, Guangzhou 510006, PR China

${ }^{2}$ Key Laboratory of Digital Quality Evaluation of Chinese Materia Medica of State Administration of TCM, Guangzhou 510006, PR China

${ }^{3}$ Engineering \& Technology Research Center for Chinese Materia Medica Quality of the Universities of Guangdong Province, Guangzhou 510006, PR China

Abstract : NaoMaiTong (NMT: Radix et Rhi-zoma Rhei, Radix Ginseng, Radix Puerariae, and Rhizoma Ligustici Chuanxiong as 9: 9: 6:6) is a traditional Chinese medicine prescription for treating ischemia cerebral apoplexy. In this work, four cell injury models of ischemic stroke were establish, namely hypoxic injury, glutamate damage, injury of potassium chloride and hydrogen peroxide damage model. The protective effects of NMT and its single herbs-containing sera of different time points on PC12 cell damage were evaluated respectively, and the corresponding efficacy-time curves were drawn. Cell viability was measured by MTT (3-(4,5)-Dimethylthiahiazo(-z-y1)-3,5-di-phenytetrazoliumromide) assay. Furthermore, an statistical methods of support vector machine (SVM) were used to establish the correlation between concentration-time-efficacy for the first time. These results revealed that NMT-containing serum has obvious protective effect on the four injury models and can significantly improve cell viability. The PK-PD correlation between fifteen ingredients in the NMT compound with four model efficacy indexes indicated that rhein, puerarin, and 3'-methoxy puerarin might be the most important 
constituents controlling the pharmacological effects of NMT. The study suggested that these fifteen components are likely to be the material basis of NMT and recommended to increase the amounts of Pueraria in the NMT compound. That provided the scientific basis and demonstration for the research of efficacy material base of Traditional Chinese Medicine (TCM).

Keywords: potential active ingredients; naomaitong; PK-PD correlation; support vector machines

\section{Introduction}

Cerebral apoplexy can be divided into ischemic cerebral apoplexy and hemorrhagic cerebral apoplexy ${ }^{[1]}$, and ischemic cerebral apoplexy accounts for eighty-five percent of cases $^{[2]}$. Cerebral apoplexy is a common clinical disease world wide and seriously endangers human health. It is characterized by its high incidence, high mortality, high recurrence rate, low cure rate and many complications. Brain tissue ischemia leading to cell death within a short time is the essence of ischemic stroke, and it irreversibly damages the brain tissue. In addition, energy depletion, increased release of excitatory amino acids, free radical damage, intracellular calcium overload, inflammatory cytokine damage, acidosis, and apoptosis-related gene activation are the main injuries that occur during ischemic cerebral apoplexy $y^{[3,4]}$. There are many research methods to investigate the treatment of cerebral ischemia, mainly including in vivo animal models and in vitro cell models ${ }^{[5]}$. According to the pathogenesis of ischemic cerebral apoplexy, we selected appropriate evaluation indicators and models to validate effective therapeutic substances. The PC12 cells line 
derived from rat adrenal pheochromocytoma cells, which is a widely used neuron-like cell model for studying neurotoxicity and neuroprotectionis currently internationally recognized in vitro neurological disease research ideal model and widely used in the studies of nervous system diseases ${ }^{[6,7]}$. It has been proven that the model of $\mathrm{Na}_{2} \mathrm{~S}_{2} \mathrm{O}_{4}$-induced hypoxia injury ${ }^{[8,9]}$ recapitulates the initial stage of injury and that Glu-induced neurotoxicity damage ${ }^{[10]}, \mathrm{KCl}$-induced calcium overload injury ${ }^{[11]}$ and $\mathrm{H}_{2} \mathrm{O}_{2}$-induced free radical damage models ${ }^{[12]}$ reflect the most serious three-steps in the process leading to cerebral ischemic injury. In China, the use of Compound Chinese Medicine (CCM) preparations to treat cerebrovascular diseases dates back to the Han Dynasty ${ }^{[13]}$. In recent years, a clinical report ${ }^{[14]}$ has shown that Chinese medicine has obvious effects and advantages for the treatment of cerebral apoplexy. The aim of the current study is to detect the effect of Nao Mai Tong on four damage models using the PC12 cells line.

NaoMaiTong (NMT), a traditional Chinese medicine prescription, consists of Rhubarb (Rheum palmatum L.), Ginseng (Panax ginseng C.A.Mey.), Ligusticum wallichii (Ligusticum chuanxiong Hort.) and Pueraria (Pueraria lobata (Willd.) Ohwi) in a ratio of 9:9:6:6 $6^{[15]}$. NMT was first described by Professor JianSheng Li (Henan University of Traditional Chinese Medicine, China) during his decades of clinical experience $^{[16]}$. In previous studies ${ }^{[14]}$, NMT has mainly been used to treat cardiovascular and cerebrovascular diseases in the clinic; it has exhibited significant therapeutic effects, which are partly ascribed to its effects on reducing brain cell damage, and has improved clinical symptoms, signs and quality of life in patients 
with cerebral infarction; it also has been demonstrated effective in treating rats with stroke, based on metabolomics ${ }^{[17]}$. In our previous study, of the 65 compounds (25 prototype compounds and 40 metabolites) in rat plasma after oral administration of NMT were detected ${ }^{[18]}$ and 15 constituents of NMT in rat plasma were predicted as the material basis based on molecular docking simulation ${ }^{[19]}$. Specifically, a comparative pharmacokinetic study of the fifteen components in rat plasma were completed $^{[15]}$. Furthermore, studies ${ }^{[16]}$ have shown that the mechanism of NMT in cerebral ischemia-reperfusion injury may be related to its ability to inhibit the expression of NF- $\mathrm{kB}, \mathrm{nNOS}$, iNOS, TNF- $\alpha, \mathrm{VCAM}-1$ and ICAM-1 ${ }^{[20]}$, increase the expression of HSP70, improve blood-brain barrier permeability, and reduce cerebral edema $^{[21]}$.

In recent years, in vitro cell models of ischemic stroke have become a popular research area. Models of reperfusion after hypoxia and hypoglycemia, glutamate injury, potassium chloride injury and hydrogen peroxide injury can successfully mimic cerebral ischemia-reperfusion injury in vivo and have been applied to pharmacological and pharmacodynamic studies of traditional Chinese medicine ${ }^{[3,4]}$, providing methodological support for the material foundation of NMT. To provide further evidence for the material basis and mechanism of action of NMT, in the present study, HPLC-MS/MS was applied to identify multi-constituents in NMT extract and its single Chinese herbs extracts. Then, we used an statistical analysis methods of SVM to establish a multi-drug efficacy index of the PK-PD ${ }^{[22]}$ correlation mathematical model for the first time. The support vector machine (SVM) algorithm 
is a powerful classification tool for creating a decision boundary between two classes that enables the prediction of labels from one or more feature vectors, which originally proposed in 1963 by Vapnik ${ }^{[23,24]}$. We synchronous systematic research the PK-PD correlation between concentration-time-effects, and the correlation between ingredients in the NMT compound with four model efficacy indexes were obtained.

\section{Results}

\subsection{Analysis of constituents of NMT extract and its single herbs extracts} HPLC-MS/MS was used for the qualitative analysis of the compound Naomaitong extract and four single herbs extract. Both the negative- and positive-ion modes were adopted for the detection of constituents in Naomaitong extract samples. In total, of the 120 constituents were identified, of which 27 components were confirmed by the reference substance. 18 components derived from rhubarb (one tannin, 14 anthraquinones and 3 diterpenoids); 51 ingredients are derived from ginseng (21 protopanaxadiol (PPD) ginsenosides, 15 protopanaxatriol (PPT) ginsenosides, 5 oleanolic acid (OA) ); 31 components from Pueraria (25 isoflavones; 2 flavonoids; 4 pueraria glycosides ); the 19 components were derived from Chuanxiong (17 phthalides, one triterpenoid, one phenolic acids). The results are shown in Fig. 1.A and Fig. 1.B. 


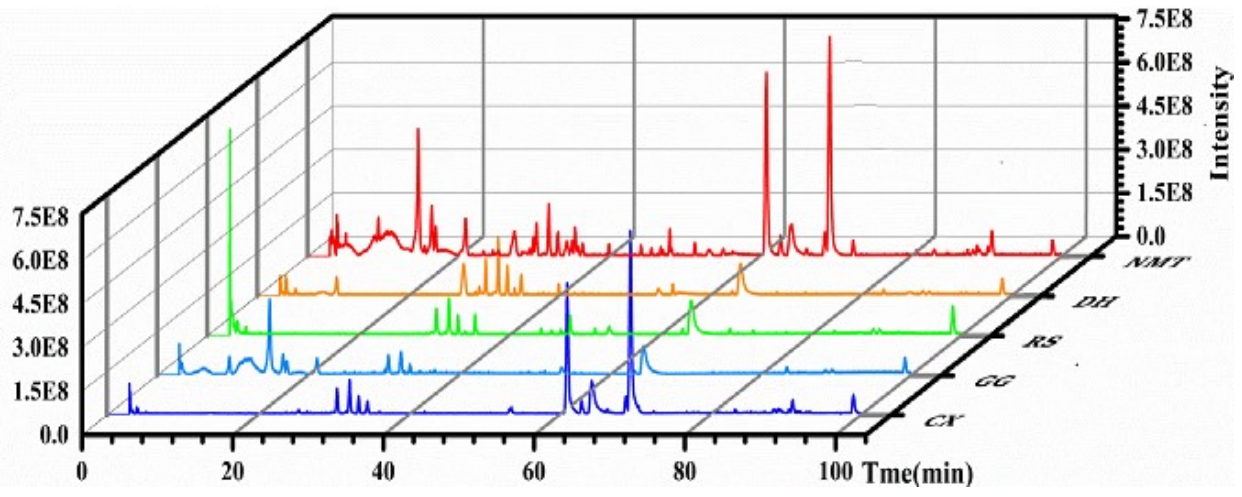

Fig. 1.A. NMT extract and its single herbs extracts base peak ion current map (in positive ion mode).

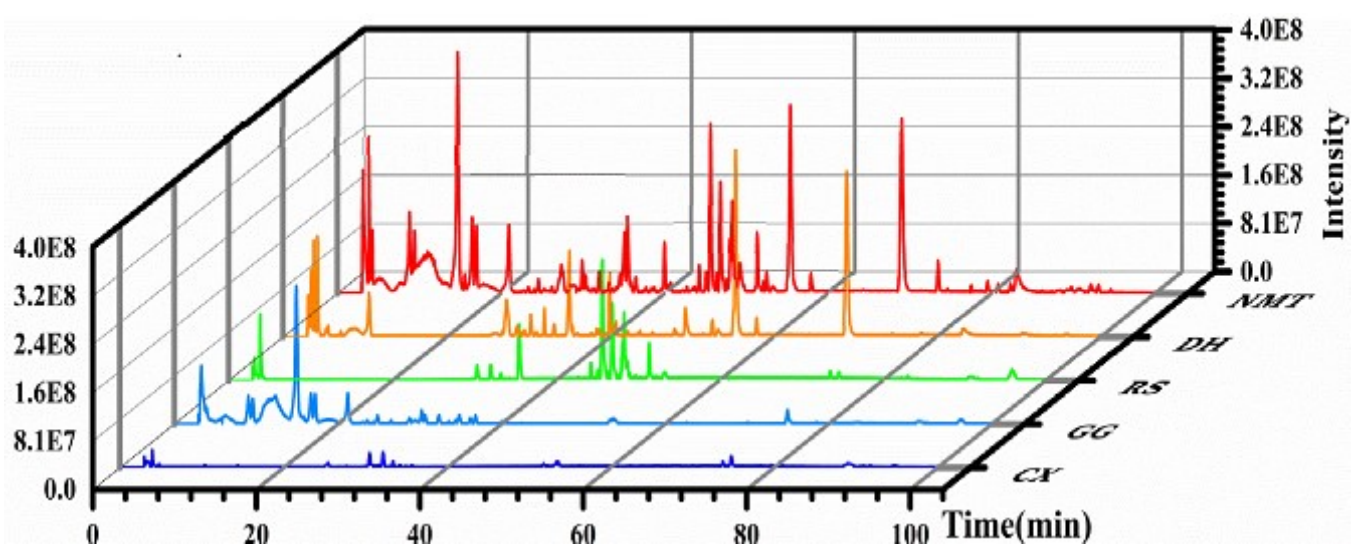

Fig. 1.B. NMT extract and its single herbs extracts base peak ion current map (in negative ion mode).

NMT: Naomaitong; DH: Rhubarb; RS: Ginseng; CX: Chuanxiong; GG: Pueraria 2.2. Protective effects of five drug-containing sera on PC12 Cell damage induced by Sodium Dithionite

Cell viability of PC12 cells, measured by MTT reduction assay. By observing the efficacy-time curve, it was found that the efficacy of NMT-containing serum reached the first peak within $0 \sim 1 \mathrm{~h}$ and the second peak within $1.5 \sim 4 \mathrm{~h}$. The first effect peak was quickly reached after 15 minutes of drug treatment, and the cell protection rate of drug-containing serum at $2 \mathrm{~h}$ was significantly higher than at $15 \mathrm{~min}$. The results 
127 showed that the cell viability increased by $10.42 \%$ and $18.99 \%$ at the first and 128 second effect peaks, respectively, compared with the control group. After $4 \mathrm{~h}$, the 129 protective effect of NMT drug-containing serum on cells suffering hypoxic injury 130 gradually decreased to $12 \mathrm{~h}$. The results are shown in Table 1 and Fig. 2. 
132 Table 1. Plasma concentration of fifteen components of Naomaitong group in normal rats (ng/mL).

\begin{tabular}{|c|c|c|c|c|c|c|c|c|c|c|c|c|c|c|c|}
\hline $\begin{array}{l}\text { time(h) } \\
\text { composition }\end{array}$ & $\begin{array}{c}\text { Emodin } \\
\text { ether } \\
\text { glycosides }\end{array}$ & $\begin{array}{l}\text { Rhubarb } \\
\text { glycosides }\end{array}$ & $\begin{array}{l}\text { Aloe } \\
\text { emodin }\end{array}$ & Chrysophanol & Rhein & Emodin & $\operatorname{Rg} 1$ & $\mathrm{Rb} 1$ & $\mathrm{Rb} 3$ & $\mathrm{Rc}$ & Puerarin & Daidzein & $\begin{array}{c}\text { 3'-methoxy } \\
\text { puerarin }\end{array}$ & Ligustilide & $\begin{array}{c}\text { Yang } \\
\text { Chuanxiong } \\
\text { lactone I }\end{array}$ \\
\hline 0 & 0.00 & 0.00 & 0.00 & 0.00 & 0.00 & 0.00 & 0.00 & 0.00 & 0.00 & 0.00 & 0.00 & 0.00 & 0.00 & 0.00 & 0.00 \\
\hline 0.083 & 33.49 & 41.45 & 40.73 & 41.39 & 415.91 & 53.66 & 2.53 & 7.43 & 3.87 & 8.31 & 149.74 & 25.66 & 531.53 & 29.19 & 44.66 \\
\hline 0.25 & 36.05 & 96.27 & 82.89 & 63.82 & 748.48 & 76.77 & 7.38 & 15.55 & 4.88 & 9.27 & 724.92 & 44.26 & 1247.43 & 173.02 & 164.12 \\
\hline 0.5 & 27.86 & 162.36 & 71.71 & 45.43 & 676.74 & 43.38 & 8.63 & 27.30 & 8.19 & 12.29 & 943.94 & 28.96 & 1218.79 & 213.51 & 122.49 \\
\hline 0.75 & 22.82 & 134.60 & 53.28 & 33.76 & 497.82 & 29.95 & 10.63 & 35.58 & 10.64 & 14.29 & 631.92 & 20.06 & 891.31 & 70.86 & 128.09 \\
\hline 1 & 23.23 & 112.66 & 38.38 & 26.83 & 419.00 & 25.82 & 13.51 & 40.07 & 16.14 & 18.91 & 480.87 & 17.49 & 771.70 & 81.02 & 130.64 \\
\hline 1.5 & 20.47 & 97.11 & 32.24 & 24.21 & 319.74 & 15.52 & 16.69 & 45.54 & 22.93 & 22.68 & 403.92 & 13.62 & 628.70 & 58.96 & 68.84 \\
\hline 2 & 15.14 & 92.81 & 17.30 & 20.78 & 267.65 & 13.30 & 20.63 & 56.31 & 30.22 & 29.27 & 254.24 & 9.64 & 457.31 & 63.47 & 30.02 \\
\hline 4 & 9.09 & 79.63 & 10.69 & 14.90 & 145.37 & 4.22 & 22.69 & 66.01 & 36.86 & 35.17 & 300.21 & 10.53 & 307.81 & 30.33 & 9.94 \\
\hline 6 & 7.10 & 45.92 & 8.27 & 15.01 & 134.98 & 5.13 & 27.26 & 79.08 & 57.02 & 47.03 & 263.36 & 14.80 & 314.15 & 35.26 & 1.99 \\
\hline 8 & 6.43 & 27.48 & 7.50 & 11.65 & 129.48 & 6.26 & 98.21 & 106.28 & 109.42 & 79.65 & 349.00 & 14.88 & 285.97 & 18.07 & 1.575 \\
\hline 12 & 5.94 & 11.54 & 4.95 & 13.48 & 191.44 & 11.18 & 36.50 & 83.51 & 36.42 & 53.40 & 248.96 & 22.89 & 344.29 & 12.30 & 1.71 \\
\hline
\end{tabular}




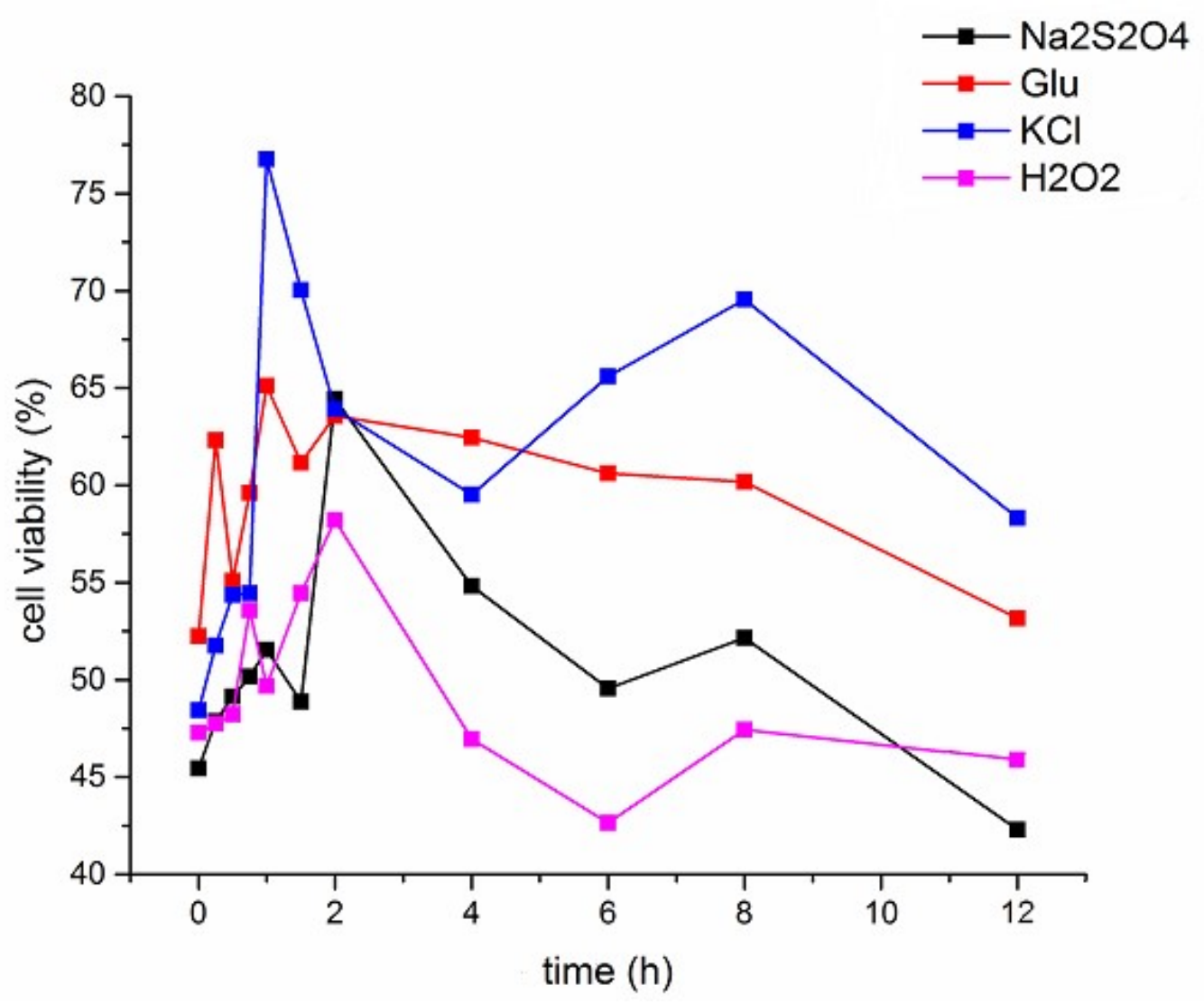

134

135

Fig. 2. The protective of efficacy-time curve of 5\% Naomaitong-containing serum of different time points on four types damage model $(\%)$.

\subsection{Protective effects of five drug-containing sera on PC12 Cell damage} induced by Glu

The cell viability of PC12 cells was, measured by MTT reduction assay. By observing the efficacy-time curve of anti-neurotoxicity damage effect, it was found that the efficacy of NMT-containing serum reached a first peak within $0 \sim 0.5 \mathrm{~h}$ and a second peak within $0.75 \mathrm{~h} \sim 1.5 \mathrm{~h}$. The first effect peak was quickly reached after 15 minutes of drug treatment, and the cell protection rate at $1 \mathrm{~h}$ was significantly higher than that at $15 \mathrm{~min}$. The results showed that the cell survival rate increased by $10.07 \%$ 
and $12.87 \%$ at the first and second peaks, respectively, compared with the control group. The anti-glutamate neurotoxic effect of NMT-containing serum had a rapid onset, and the protection rate was maintained at a high level for $8 \mathrm{~h}$ and gradually decreased after $12 \mathrm{~h}$. The results are shown in Table 1 and Fig. 2.

\subsection{Protective effects of five drug-containing sera on PC12 Cell damage} induced by Potassium chloride

Cell viability of PC12 cells, was measured by MTT reduction assay. By observing the efficacy-time curve of the anti-calcium overload injury effect, it was found that the efficacy of NMT-containing serum reached a first peak within $0.5 \sim 2 \mathrm{~h}$ and a second peak within $4 \mathrm{~h} \sim 12 \mathrm{~h}$. The cell protection rate at the first effect peak at 1 $\mathrm{h}$ was slightly higher than that at the second peak at $8 \mathrm{~h}$. The results showed that the cell survival rate increased by $28.32 \%$ and $21.12 \%$ at the first and second peaks, respectively, compared with the control group. It is worth noting that the drug worked slightly less rapidly until $1 \mathrm{~h}$. The protection rate was maintained at a high level over $12 \mathrm{~h}$ and a weak protective effect persisted after $12 \mathrm{~h}$. The results are shown in Table 1 and Fig. 2.

2.5. Protective effects of five drug-containing sera on PC12 Cell damage induced by Hydrogen peroxide

Cell viability of PC12 cells, was measured by MTT reduction assay. By observing the efficacy-time curves of the anti-free radical damage effect, it was found that the efficacy of NMT-containing serum reached a first peak within $0.5 \sim 1 \mathrm{~h}$ and a second peak within $1.5 \mathrm{~h} \sim 4 \mathrm{~h}$. The cell protection rate at the first effect peak at $0.75 \mathrm{~h}$ 
was slightly higher than that at the second peak at $2 \mathrm{~h}$. However, the results showed that the cell survival rates at the two effect peaks were not obviousiy increased compared with the control group. In addition, NMT-containing serum had no protective effect against free radical damage after $4 \mathrm{~h}$. The results are shown in Table 1 and Fig. 2.

\subsection{PK and PD data analysis}

As shown in Table 2, UPLC-MS was used to study the pharmacokinetics of the Naomaitong serum used in the cell pharmacodynamics experiments to obtain the concentration-time curves of each component of NMT in serum. Each herbal ingredient in serum has a different pattern of efficacy-time curves for each cell injury model. As shown in Table 6, the results reflect their own unique relationship between cell viability and time. The PD-PK binding model can be used study the relationship between the concentration, time and synergistic effects of multiple components ${ }^{[25,26]}$.

The results are shown in Fig. 3 6.

Table 2 .Cell viability of four types damage model protected by Naomaitong group drug-containing serum (\%).

\begin{tabular}{|c|c|c|c|c|}
\hline time $(\mathrm{h})$ & $\mathrm{Na}_{2} \mathrm{~S}_{2} \mathrm{O}_{4}$ & Glu & $\mathrm{KCl}$ & $\mathrm{H}_{2} \mathrm{O}_{2}$ \\
\hline group & Model group & Model group & Model group & Model group \\
\hline 0 & 45.44 & 52.24 & 48.44 & 47.28 \\
\hline 0.083 & 46.40 & 58.45 & 49.68 & 49.37 \\
\hline 0.25 & 47.86 & 62.31 & 51.76 & 47.73 \\
\hline 0.5 & 49.14 & 55.08 & 54.36 & 48.22 \\
\hline 0.75 & 50.17 & 59.61 & 54.44 & 53.58 \\
\hline 1 & 51.54 & 65.11 & 76.76 & 49.69 \\
\hline 1.5 & 48.87 & 61.15 & 70.04 & 54.44 \\
\hline
\end{tabular}




\begin{tabular}{ccccc}
2 & 64.43 & 63.57 & 63.92 & 58.21 \\
4 & 54.83 & 62.45 & 59.52 & 46.95 \\
6 & 49.55 & 60.62 & 65.60 & 42.65 \\
8 & 52.16 & 60.17 & 69.56 & 47.44 \\
12 & 42.29 & 53.16 & 58.32 & 45.89 \\
\hline
\end{tabular}

184

185

186

187

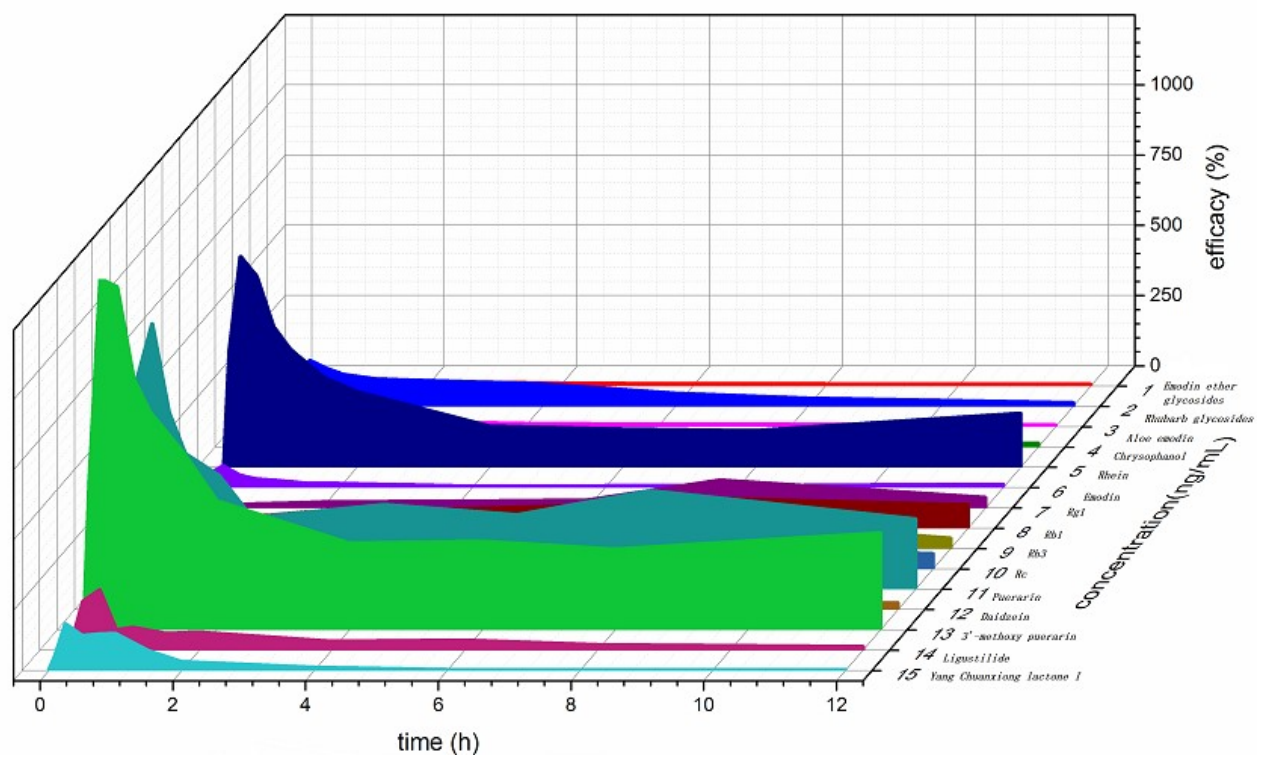

Fig. 3. Three-dimension graph of time-concentration-effect of $\mathrm{Na}_{2} \mathrm{~S}_{2} \mathrm{O}_{4}$ damage model based on PK-PD modeling.

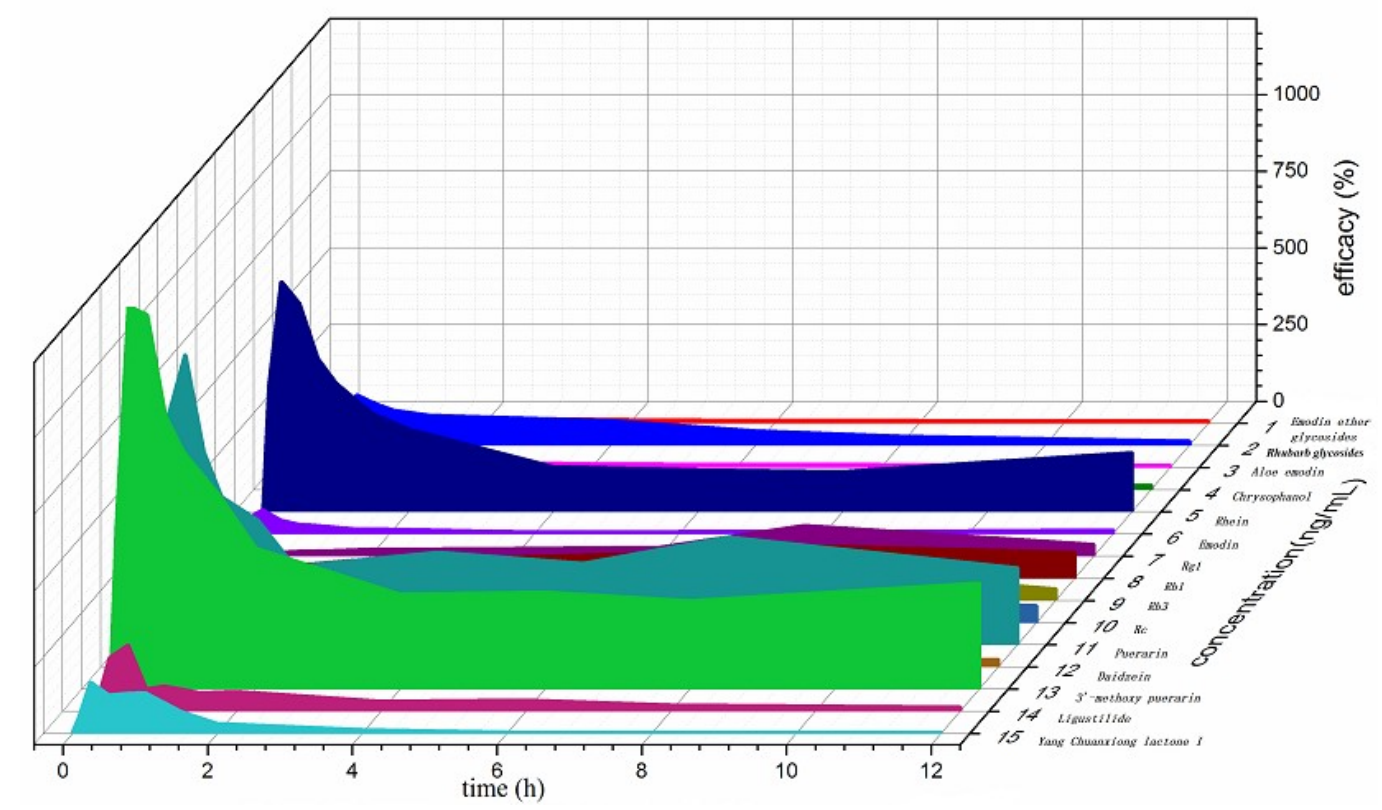


189

190

191

192

193

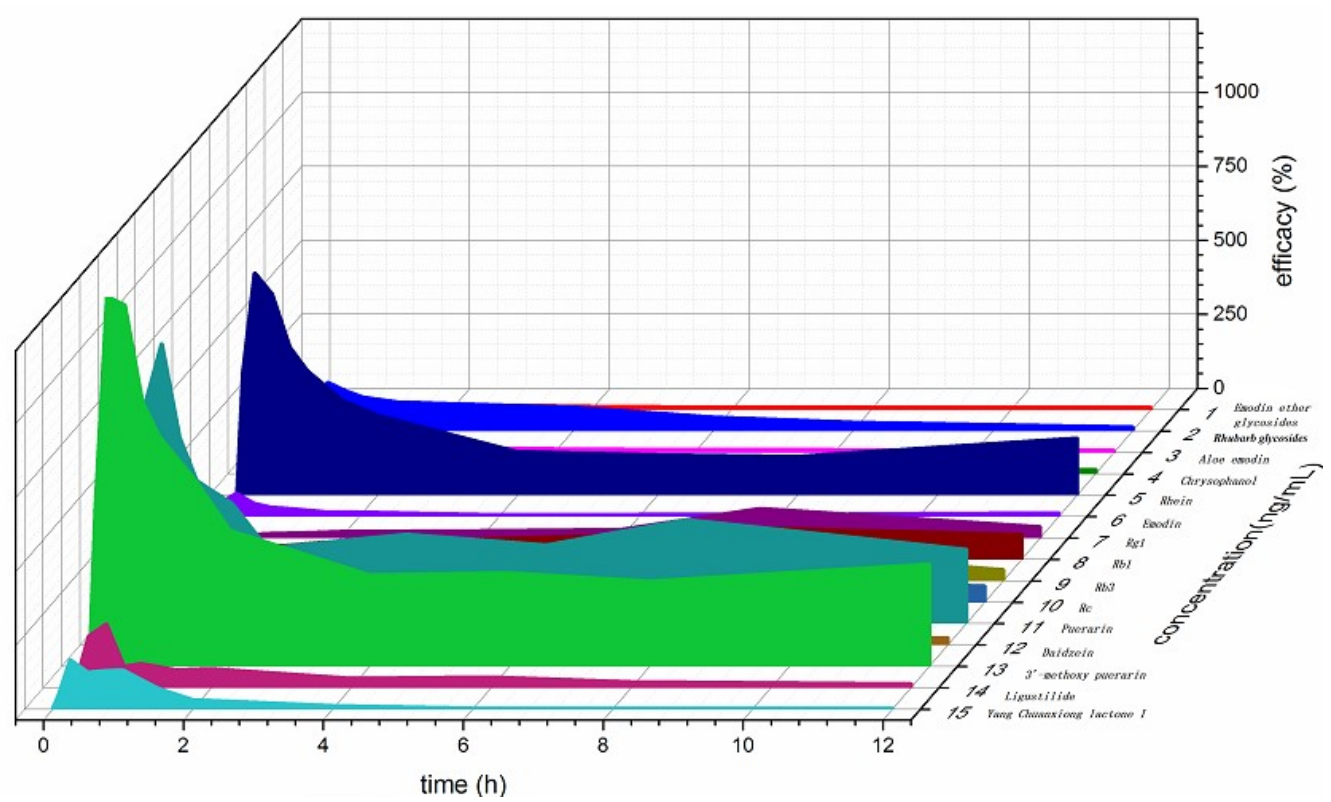

194

195

196

197

198

199

Fig. 4. Three-dimension graph of time-concentration-effect of Glu damage model based on PK-PD modeling. 


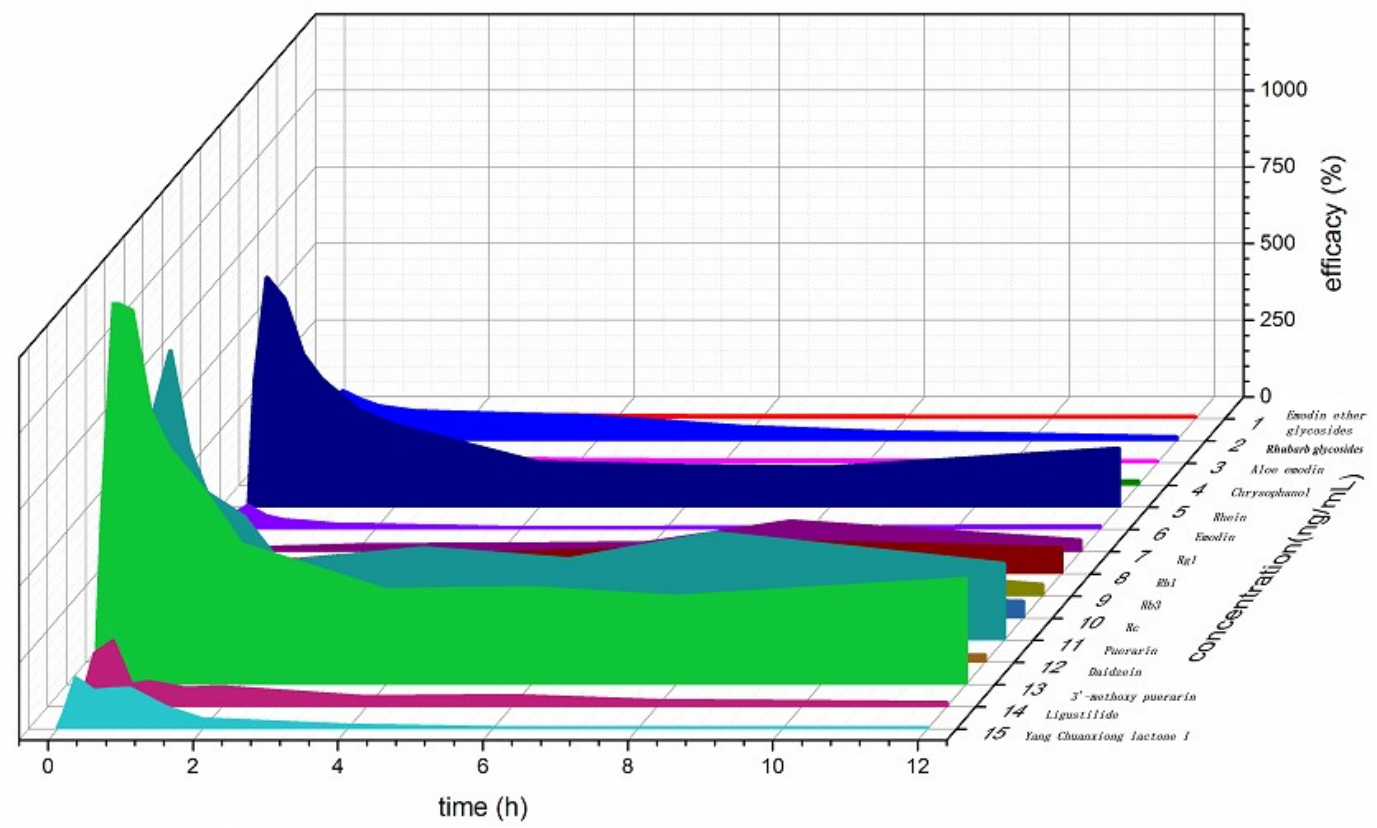

201 Fig. 6. Three-dimension graph of time-concentration-effect of $\mathrm{H}_{2} \mathrm{O}_{2}$ damage model based on PK-PD modeling.

\subsection{SVM for PK-PD Correlation Study}

After running the SVM program, the weight coefficient $w$ of each component for each model pharmacokinetic index was calculated, and the weights of the fifteen components on the efficacy can be directly seen in the table. According to the weight coefficient $w$, the correlation between each component and each model index can be analyzed. The results are shown in Table 3. 
210 Table 3. The weight coefficient $w$ of each component to the four models by SVM used in the PK - PD correlation study.

211

\begin{tabular}{|c|c|c|c|c|c|c|c|c|c|c|c|c|c|c|c|}
\hline $\begin{array}{c}\text { group } \\
\text { Composition }\end{array}$ & $\begin{array}{c}\text { 3'-methoxy } \\
\text { puerarin }\end{array}$ & Puerarin & Rhein & $\begin{array}{l}\text { Rhubarb } \\
\text { glycosides }\end{array}$ & Ligustilide & $\begin{array}{c}\text { Yang } \\
\text { Chuanxiong } \\
\text { lactone I }\end{array}$ & $\begin{array}{c}\text { Aloe } \\
\text { emodin }\end{array}$ & $\begin{array}{l}\text { Chryso } \\
\text { phanol }\end{array}$ & $\begin{array}{c}\text { Emodin } \\
\text { ether } \\
\text { glycosides }\end{array}$ & $\operatorname{Rg} 1$ & Emodin & Daidzein & $\mathrm{Rb} 1$ & $\mathrm{Rc}$ & Rb3 \\
\hline \multicolumn{16}{|l|}{$\mathrm{Na}_{2} \mathrm{~S}_{2} \mathrm{O}_{4}$} \\
\hline $\begin{array}{l}\text { Model } \\
\text { group }\end{array}$ & 19.6494 & 8.3769 & 4.7835 & 2.3775 & 1.6930 & 0.8312 & 0.4441 & 0.3337 & 0.2471 & 0.1414 & 0.1081 & 0.0831 & -1.2304 & -1.2350 & -1.3253 \\
\hline \multicolumn{16}{|l|}{ Glu } \\
\hline $\begin{array}{l}\text { Model } \\
\text { group }\end{array}$ & 13.4528 & 5.7142 & 3.5973 & 1.9712 & 0.7771 & 0.7927 & 0.2689 & 0.2736 & 0.2734 & 0.4087 & 0.2538 & 0.1301 & 1.7844 & -0.1875 & -0.1617 \\
\hline \multicolumn{16}{|l|}{$\mathrm{KCl}$} \\
\hline $\begin{array}{l}\text { Model } \\
\text { group }\end{array}$ & 10.9959 & 4.7927 & 5.1024 & 2.2129 & -0.7450 & 0.0121 & -0.5323 & -0.4758 & -0.1793 & 0.8945 & -1.5406 & -0.1616 & 14.3841 & 5.4839 & 3.3204 \\
\hline \multicolumn{16}{|l|}{$\mathrm{H}_{2} \mathrm{O}_{2}$} \\
\hline $\begin{array}{l}\text { Model } \\
\text { group }\end{array}$ & 18.4822 & 7.8900 & 5.4022 & 2.1043 & 0.9089 & 0.8333 & 0.1806 & 0.2298 & 0.2962 & 0.0836 & 0.3046 & 0.0689 & -1.3702 & -1.5871 & -1.1441 \\
\hline
\end{tabular}




\section{Discussion}

By observing the efficacy-time curves of the NMT-containing sera on the protective effects in four cell injury models, it was found that the drug-containing sera showed different efficacy-time relationships for different models. For the $\mathrm{Na}_{2} \mathrm{~S}_{2} \mathrm{O}_{4}$ injury model, there are two effect peaks, and the second peak was significantly higher than the first peak. For the Glu injury model, there are two similar effect peaks in the efficacy-time curve, and the cytoprotection curves of the drug-containing sera in this model showed a longer protection duration. For the $\mathrm{KCl}$ injury model, the protective effect of drug-containing sera showed a slow onset, with the first effect peak at $1 \mathrm{~h}$,

222 followed by reduced efficacy but a sustained high cytoprotective effect. For the $\mathrm{H}_{2} \mathrm{O}_{2}$ 223 injury model, the efficacy-time curve of the drug-containing serum showed two weaker effect peaks with a relatively weaker cytoprotective effect. In conclusion, the NMT-containing serum at different time points showed the most significant effect on the $\mathrm{KCl}$-induced injury model, followed by an obvious protective effect on Glu- and $\mathrm{Na}_{2} \mathrm{~S}_{2} \mathrm{O}_{4}$-induced injury models, and $\mathrm{H}_{2} \mathrm{O}_{2}$-induced injury, although the protective effect against $\mathrm{H}_{2} \mathrm{O}_{2}$-induced injury was not obvious compared to the effects on the other three models. time points, it was found that rhubarb-containing serum has a strong cytoprotective effect on Glu and $\mathrm{H}_{2} \mathrm{O}_{2}$ injury models, while the protective effect in $\mathrm{Na}_{2} \mathrm{~S}_{2} \mathrm{O}_{4}$ and $\mathrm{KCl}$ injury models was weak. This indicated that rhubarb has a better protective effect on 
glutamate neurotoxicity and oxidative stress injury in cerebral ischemia. By comparing the efficacy-time curves of ginseng-containing serum in different injury models, it was found that ginseng-containing serum had a stronger protective effect on the $\mathrm{KCl}$ damage model. For the other three injury models, the pharmacodynamic effects were not significant and were lower than those observed in the $\mathrm{KCl}$ damage model. Our data show that Ginseng mainly has an obvious protective effect on intracellular calcium overload damage of cerebral ischemia.In the efficacy-time curve of the protective effect of Pueraria-containing serum on the $\mathrm{Na}_{2} \mathrm{~S}_{2} \mathrm{O}_{4}$ damage model, there are three effect peaks; The drug-containing serum has a rapid onset in protecting against $\mathrm{H}_{2} \mathrm{O}_{2}$ injury, and its efficacy is maintained at a high level within $2 \mathrm{~h}$. However, the protective effect of Pueraria-containing serum against Glu and $\mathrm{KCl}$ injury is not obvious. It is speculated that puerarin has a better pharmacological effect on ischemic hypoxia and oxidative stress injury in cerebral ischemia. By comparing the efficacy-time curves of Chuanxiong-containing serum with different injury models, it was found that the protective effects of the serum against $\mathrm{Na}_{2} \mathrm{~S}_{2} \mathrm{O}_{4}$ and $\mathrm{H}_{2} \mathrm{O}_{2}$ damage were both obvious, while the efficacy was not obvious for Glu and $\mathrm{KCl}$ damage.It is speculated that Chuanxiong has a better pharmacological effect on the energy failure and oxidative stress injury in cerebral ischemia.

A comparison of the efficacy-time curves of the compound and the four single herbs showed that each single herb has obvious protective effects in only one or two damage models, while the compound has an obvious protective effect on the four mechanism damage models. The analysis of the overall pharmacodynamic effect 
indicates that the effect of the NMT compound was significantly better than those of

257 the single herbs in the four types of in vitro cell injury models of ischemic cerebral 258 apoplexy, consistent with the "multi-component, multi-channel, multi-target" 259 synergistic effects that exist in Traditional Chinese Medicine (TCM). These results

could be reasonably speculated to be in accordance with the qualities of integrality, multiple-effects and complexity that are characteristic of traditional Chinese medicine compounds. In view of the different mechanisms of the injury models, the drug-containing sera showed different relationships of efficacy and time in PC12 cells, which reflects the "Jun-Chen-Zuo-Shi" rule of NMT from the perspective of cellular pharmacodynamics and provides a reference for further study of the material basis of the compound NMT.

The main injury processes involved in ischemic stroke include energy depletion, increased release of excitatory amino acids, free radical damage and intracellular calcium overload ${ }^{[3,4]}$. These injuries affect, overlap and correlate with each other and eventually lead to the death of nerve cells. Comprehensive analysis of the efficacy-time curves revealed that the drug-containing serum showed a more significant protective effect on $\mathrm{Na}_{2} \mathrm{~S}_{2} \mathrm{O}_{4}$ and Glu injury within $0 \sim 0.5 \mathrm{~h}$, indicating its rapid onset; this shows that its pharmacodynamic effects may be exerted mainly during a short period of time to ameliorate energy failure and glutamate neurotoxicity. In addition, the drug-containing serum has a good protective effect in all four models within $0.75 \sim 2 \mathrm{~h}$, and the efficacy is higher at this time than at $0 \sim 0.5 \mathrm{~h}$, indicating that the pharmacodynamic effects of NMT may have multiple targets during this time. 
NMT can not only improve energy failure and damage due to glutamate neurotoxicity but also reduce the damage caused by calcium ion overload and excessive free radicals. The protective effect of drug-containing serum within $4 \sim 24 \mathrm{~h}$ began to decline gradually, except for the maximum effect peak at $8 \mathrm{~h}$ in the $\mathrm{KCl}$ model, indicating that in the later stages, NMT exerts its effects mainly by reducing the damage caused by calcium overload. Four types of cell injury models were used to simulate different injury mechanisms of ischemic stroke. The pharmacodynamic effects of sera containing NMT and its single constituent herbs on various cell injury models were comprehensively studied which provided an effective basis to study the correlation between the PK-PD of the multiple components of NMT. The PD-PK binding model can be used to study the relationship between the concentration-time-effect of organic integration, thereby revealing the synergistic or antagonistic interactions between the chemical components of prescriptions and ascertaining the material basis of TCM prescriptions ${ }^{[27,28]}$.

By using SVM to establish the PK-PD binding model of NMT serum, it was possible to carry out a correlation analysis to determine the material basis of NMT. Previous studies have shown that there are significant pharmacodynamic effects of individual components such as rhubarb glycosides, emodin, chrysophanol, emodin ether, rhein ${ }^{[29-31]}$ and so on in the treatment of cerebral ischemia. It has also been shown that monomer components such as the ginsenosides $\mathrm{Rg} 1, \mathrm{Rb} 1, \mathrm{Rb} 3$ and $\mathrm{Rd}^{[32,33]}$ were significant in cerebral ischemia; the effects of the Pueraria isoflavones puerarin, $3^{\prime}$-methoxy puerarin, daidzein ${ }^{[34,35]}$ and other components of anti-cerebral ischemia 
300

301

treatments are significant; and Chuanxiong volatile oil ${ }^{[36,37]}$ can play a protective role in cerebral ischemia. There are fifteen known active ingredients in NMT-containing serum, including rhubarb glycosides, emodin ether glycosides, rhein, aloe emodin, emodin, chrysophanol, ginsenoside Rb3, Rb1, Rg1, Rc, daidzein, 3'-methoxy puerarin, puerarin, Yang Chuanxiong lactone I and ligustilide, which have a good correlation with the four cell models of cerebral ischemia and may be the effect components of NMT. The efficacy of some of these ingredients has been demonstrated by studies, and others remain to be validated. In a comparison of the results of the ANN and SVM methods, the results showed that SVM has a great advantage in dealing with the nonlinear relationships in experiments with small sample sizes, and our comparison indicated that SVM is more suitable for PK-PD correlation studies with few samples. According to the PK-PD fitting results of SVM, for different models, it was found that the weights of the ingredients on the efficacy indicators are not the same, and they also show different positive and negative correlations. For the $\mathrm{Na}_{2} \mathrm{~S}_{2} \mathrm{O}_{4}$ injury model, in addition to $\mathrm{Rb} 1, \mathrm{Rc}$, and $\mathrm{Rb} 3$, the remaining components were positively correlated with drug efficacy, and these components may be the main pharmacodynamic components. For the Glu injury model, with the exception of Rb3 and Rc, the remaining components were positively correlated with drug efficacy. For the $\mathrm{KCl}$ injury model, the components which positively related to the drug efficacy are Rb1, 3'-methoxy puerarin, Rc, rhein, puerarin, Rb3, rhubarb glycosides, Rg1, and Yang Chuanxiong lactone I. These nine components may be the main pharmacodynamic components that works against intracellular calcium overload 
damage. For the $\mathrm{H}_{2} \mathrm{O}_{2}$ injury model, with the exception of $\mathrm{Rb} 3, \mathrm{Rb} 1$, and $\mathrm{Rc}$, the rest of components were positively correlated with drug efficacy, and may be the main medicinal ingredients for protecting against oxidative stress injury.

In this study, the statistical analysis methods SVM was used for the first time to study the material basis of the effectiveness of NMT. The correlation of PK-PD between fifteen components in the compound and the four models indicates that these fifteen components are likely to be the NMT effect substances. This finding provides a scientific basis and demonstration of basic research into Chinese medicine compound pharmacodynamics. It is worth mentioning that Pueraria-containing serum had three effect peaks in cells suffering hypoxic injury, and the cell viability at $1.5 \mathrm{~h}$ increased by $10.51 \%$ compared with that of the control group, while NMT-containing serum had two effect peaks, and the cell viability at $0.25 \mathrm{~h}$ increased by $10.42 \%$. The Chuanxiong-containing serum had three effect peaks in cells suffering from Glu damage, and the cell viability at $1 \mathrm{~h}$ increased by $20.71 \%$ compared with that of the control group, while NMT-containing serum had two effect peaks and the cell viability at $1 \mathrm{~h}$ increased by only $12.87 \%$. Moreover, the PK-PD correlation study shown that pharmacodynamic indicators of rhein, puerarin, and 3'-methoxy puerarin are significant. Therefore, it is recommended to increase the amounts of Pueraria and Chuanxiong in the NMT compound in order to target treatment. In summary, due to the complexity of the traditional Chinese medicine compound, the reasons for the above results and its specific mechanism of action are yet to be studied. To further support our finding, the specific pharmacological mechanism of action of each 
monomer component remains to be studied. The pharmacodynamics of rhein, puerarin, 3'-methoxy puerarin and NMT can be further validated based on the rat focal cerebral ischemia models which were prepared according to the modified method of Longa ${ }^{[38]}$.

\section{Materials and Methods}

\subsection{Ethics statement}

All procedures had the approval of the Animal Ethics Committee of the Guangdong Pharmaceutical University.

\subsection{Chemicals and Reagents}

Sodium hyposulfite was purchased from Tianjin Damao Chemical Reagent Factory (Tianjin, China). Glutamic acid was prepared by Sinopharm Chemical Reagent Co., Ltd. (Shanghai, China). Potassium chloride was purchased from Tianjin Zhiyuan Chemical Reagent Co., Ltd. (Tianjin, China). Hydrogen peroxide (30, \%), was obtained from Guangzhou Chemical Reagent Factory (Guangzhou, China). RPMI-1640 medium and trypsin were provided by HyClone (Logan, Utah, USA). 3-(4,5)-Dimethylthiahiazo(-z-y1)-3,5-di-phenytetrazoliumromide-MTT and dimethyl sulfoxide were obtained from Amresco (Radnor, Pennsylvania, USA). Natural bovine calf serum was purchased from Gibco (California, USA).

\subsection{Plant Material}

Rhubarb (the root of Rheum palmatum L., batch number: 140,310), Ginseng (the root of Panax ginseng C.A. Mey., batch number:140,225), Pueraria (the root of Puer-aria lobata (Willd.) Ohwi, batch number:140,114), and Ligusticum wallichii (the root of Ligusticum chuanxiong hort, batch number:140,301) were purchased from a 
Chinese herbal medicine market (Bozhou, Anhui, China) and were identified by Li Shuyuan, Professor of Traditional Chinese Medicine at Guangdong Pharmaceutical University.

\subsection{Cell culture}

Well-differentiated PC12 cells provided by the Experimental Center at the School of Medicine, Sun Yat-sen University, were grown in $25 \mathrm{~cm}^{2}$ polystyrene flasks with $10 \%$ calf serum in RPMI-1640 medium. The cultures were maintained at $37{ }^{\circ} \mathrm{C}$ in a humidified atmosphere containing $95 \%$ air and $5 \% \mathrm{CO}_{2}$. The culture medium was replaced every $2 \mathrm{~d}$, and cultures were split at a ratio of $1: 2$ or $1: 3$ each time. The cells were seeded at an appropri-ate density $\left(5 \times 10^{6}\right.$ cells $\left./ \mathrm{mL}\right)$ in culture dishes and were inoculated onto 96-well plates when grown to anastomosis. Prior to experiments, cell viability was assessed by trypan blue dye exclusiontest, and only cell batches showing $>95 \%$ viability were used in the study ${ }^{[39]}$.

\subsection{Preparation of liquid medicine}

NMT ( rhubarb: ginseng: puerarin: chuanxiong $=9: 9: 6: 6$ by mass weight ) was immersed in a tenfold excess (based on mass weight) of $60 \%$ ethanol-water solution ${ }^{[40]}$ and extracted by refluxing twice at $80^{\circ} \mathrm{C}$, one hour each time. The extracts were filtered separately, and then the two filtrates were combined and concentrated to $0.5 \mathrm{~g} / \mathrm{mL}$ sample drug. The concentrated solutions of Radix et Rhizoma Rhei, Panax gin-seng C.A. Mey., Pueraria lobata (Willd.) Ohwi., and Ligus-ticum chuanxiong Hort. were obtained in the same way.

\subsection{Instrument and chromatographic conditions}


$30^{\circ} \mathrm{C}$ by passing through the mobile phase, which was composed of acetonitrile (A) and $0.1 \%$ for-mic acid in water (B). The gradient conditions of the mobile phase were as follows: $0 \sim 5 \mathrm{~min}, 10 \% \mathrm{~A}$; 5 18 $\mathrm{min}, 10 \% \sim 14 \% \mathrm{~A} ; 18 \sim 25 \mathrm{~min}, 14 \% \sim 20 \% \mathrm{~A}$; 25 35 $\min , 20 \% \sim 30 \%$ A; 35 50 $\min , 30 \% \sim 40 \%$ A; 50 90 $\min , 40 \% \sim 66 \%$ A; 90 95 $\min , 66 \% \sim 70 \% \mathrm{~A} ; 95 \sim 100 \mathrm{~min}, 70 \% \mathrm{~A}$. The flow rate was maintained at $1 \mathrm{~mL} \mathrm{~min}{ }^{-1}$ and the injection volume was $10 \mu \mathrm{L}$.

A high-resolution Q-Exactive mass spectrometer (Thermo Fisher Scientific, Bremen, Germany), specifi-cally a quadrupole-orbitrap hybrid mass spectrometer cou-pled with a heated electrospray ionization (HESI) source, was operated in both positive and negative ion modes. The mass conditions were as follows: spray volt-age,

$\mathrm{L} \mathrm{min}^{-1}$; auxiliary gas heater temperature, $200{ }^{\circ} \mathrm{Cscan}$ range, $\mathrm{m} / \mathrm{z} 100-\mathrm{m} / \mathrm{z} 1500$; stepped NCE, 20, 35 eV. XCalibur 3.0 soft-ware (Thermo Fisher Scientific, USA) was used to control the instrument and for data acquisition and analysis. Q-Exactive 2.0

\subsection{Animal Experiments and Blood Sample Extraction}

The animal welfare and experimental procedures were strictly performed Laboratory Animals of China. Thirty adult Sprague-Dawley rats, weighing 280-300 g, 
were purchased from the Guangzhou University of Traditional Chinese Medicine Laboratory Animal Center (Guangzhou, China) , PRC (Certificate no. SCXK 2013-0020) ${ }^{[15]}$ and maintained in a 12-h light/12-h dark cycle and a temperature of $20 \pm 2{ }^{\circ} \mathrm{C}$ with a humidity of $50 \pm 10 \%$ for seven days before the experiments ${ }^{[18]}$. All rats were randomly divided into six groups( $\mathrm{n}=6$ for each group). One group was selected as control group and administration with saline, and the rest were marked as the single-drug groups (Da Huang, Ren Shen, Ge Gen, and Chuan Xiong) and the NMT group. The experimental groups were administered single drug concentrate solutions (12 g/kg) or NMT (12 g/kg) once per day for three days; the amount of drug administered to the single-drug groups and the single herb components of the compound control group were the same. All rats were fasted but allowed water for 12 hours until the last gavage. After the last administration, the drug-containing serum samples were collected from the fundus venous plexus at $0 \mathrm{~min}, 5 \mathrm{~min}, 15 \mathrm{~min}, 30$ min, 45 min, 1 h, 1.5 h, 2 h, 4 h, 6 h, 8 h, 12 h and 24 h. Serum was collected from the blank control group in the same way. Next, the serum samples were left to stand for 2 hours and centrifuged at $4000 \mathrm{rpm}$ for $15 \mathrm{~min}$.Then, the serum of each group was combined and immersed in a $56{ }^{\circ} \mathrm{C}$ water bath for $30 \mathrm{~min}$, filtered with a $0.22 \mu \mathrm{m}$ microporous membrane and stored at $-80{ }^{\circ} \mathrm{C}$ until analysis. All protocols and rat care was performed in accordance with the relevant national legislation and local guidelines. 

to logarithmic growth phase. All cells were randomly divided into fourteen groups.

434 Each group was inoculated into four wells, and RPMI-1640 culture solution 435 containing $5 \%$ corresponding serum was added. The groupings were as follows: (1) 436 blank control group; (2) model control group; (3) (14): NMT-containing serum 437 groups. Groups (1) and (2) were incubated with RPMI-1640 medium containing 5\% blank serum, while groups (3) (14) were incubated with RPMI-1640 medium containing $5 \%$ of the corresponding drug-containing serum at different time points and preprotected for $2 \mathrm{~h}$. In addition to the blank control group, groups (2) (14) were treated with $\mathrm{Na}_{2} \mathrm{~S}_{2} \mathrm{O}_{4}$ solution to a final concentration of $15 \mathrm{mmol} / \mathrm{L}$ for $4 \mathrm{~h}$ to induce PC12 cell damage. Subsequently, the original cell culture medium containing $\mathrm{Na}_{2} \mathrm{~S}_{2} \mathrm{O}_{4}$ solution was removed, and RPMI-1640 medium without calf serum was added to every well. The cells were maintained at $37^{\circ} \mathrm{C}$ in a humidified atmosphere containing $5 \% \mathrm{CO}_{2}$ for $24 \mathrm{~h}$. Cell viability was determined by MTT reduction assay, and the

446 efficacy-time curves were plotted. The experiment was repeated three times.

447 4.9. Protective effects of five drug-containing sera against Glutamate-induced neurotoxicity damage in PC12 Cells control group, groups (2) (14) were treated with Glu solution to a final concentration of $25 \mathrm{mmol} / \mathrm{L}$ for $1 \mathrm{~h}$ to induce neurotoxicity damage. Subsequently, the original cell culture medium containing Glu solution was removed, and RPMI-1640 medium 
without calf serum was added to every well. The cells were maintained at $37^{\circ} \mathrm{C}$ in a humidified atmosphere containing $5 \% \mathrm{CO}_{2}$ for $24 \mathrm{~h}$. Cell viability was determined by MTT reduction assay, and the efficacy-time curve was plotted. The experiment was repeated three times.

4.10. Protective effects of five drug-containing sera against Potassium chloride -induced calcium overload injury in PC12 Cells

The groupings in this experiment are the same as those described above. After all drug-containing serum groups were preprotected for $12 \mathrm{~h}$, in addition to the blank control group, groups (2) (14) were treated with $\mathrm{KCl}$ solution to the final concentration of $75 \mathrm{mmol} / \mathrm{L}$ for $4 \mathrm{~h}$ to induce calcium overload damage. Subsequently, the original cell culture medium containing $\mathrm{KCl}$ solution was removed, and RPMI-1640 medium without calf serum was added to every well. The cells were maintained at $37^{\circ} \mathrm{C}$ in a humidified atmosphere containing $5 \% \mathrm{CO}_{2}$ for $24 \mathrm{~h}$. Cell viability was determined by MTT reduction assay, and the efficacy-time curve was plotted. The experiment was repeated three times.

\subsection{Protective effects of five drug-containing sera against Hydrogen} peroxide-induced free radical damage in PC12 Cells

The groupings in this experiment are the same as those described above. After all drug-containing serum groups were preprotected for $12 \mathrm{~h}$, in addition to the blank control group, groups (2) (14) were supplemented with $\mathrm{H}_{2} \mathrm{O}_{2}$ solution to a the concentration of $150 \mu \mathrm{mol} / \mathrm{L}$ for $2 \mathrm{~h}$ to induce free radical damage in PC12 cells. Subsequently, the original cell culture medium containing $\mathrm{H}_{2} \mathrm{O}_{2}$ solution was removed, 
and RPMI-1640 medium without calf serum was added to every well. The cells were maintained at $37^{\circ} \mathrm{C}$ in a humidified atmosphere containing $5 \% \mathrm{CO}_{2}$ for $24 \mathrm{~h}$. Cell viability was determined by MTT reduction assay, and the efficacy-time curve was plotted. The experiment was repeated three times.

\subsection{Measurement of Cell Viability}

Cell viability was assessed using the MTT assay. The cells were seeded on 96-well plates with $5 \times 10^{6}$ cells in $200 \mu \mathrm{L}$ medium per well and cultured $24 \mathrm{~h}$ for stabilization. At the end of the exposure time, MTT solution $(5 \mathrm{mg} / \mathrm{mL})$ was added to each well to a final concentration of $0.5 \mathrm{mg} / \mathrm{ml}$, and the cells were cultured for $4 \mathrm{~h}$ at $37^{\circ} \mathrm{C}^{[41]}$. The medium was then removed carefully and $150 \mu 1$ DMSO was added and mixed with the cells thoroughly until the formazan crystals were dissolved completely. The resulting solution was measured in a microplate reader (iMark, Bio-Rad, USA) at a wavelength of $490 \mathrm{~nm}$. The experiment groups were divided into a zero setting (blank) group, control group, and experimental group ${ }^{[42]}$. Cell viability was expressed as a percentage of the viability of the control culture. Meanwhile, the viability in the rest of the injury groups was assessed according to the following formula:

Cell survival rate $(\%)=[\mathrm{OD}$ (Drug-containing serum group) - OD (blank) $] /[\mathrm{OD}$ (untreated control group) - OD (blank)] × 100\%(1)

\subsection{Statistical analysis}

\subsubsection{Establish the efficacy-time curve}

The results were expressed as the mean and standard deviation $(\mathrm{x} \pm \mathrm{s})$. One-way ANOVA was performed for comparisons of multiple groups, and significance was 
accepted at $p<0.05$. The efficacy-time curves of the compound and its single herbs on each cell model were drawn using pharmacokinetics software.

\subsubsection{Calculate the PK and PD data}

Pharmacokinetics software (PKSolver software) was used to calculate the blood concentration of each component index parameter (according to the individual components of each final herbal medicine). Pharmacodynamic data for the NMT-containing serum were obtained in the previous experiment, and the experimental results were compiled in origin 9.0 software to obtain the change in cell viability of the injury model over time. The multi-drug efficacy index of the PK-PD correlation mathematical model was established using origin 9.0 software.

\subsubsection{An statistical analysis methods: SVM}

SVM is a learning method that specializes in finite sample prediction ${ }^{[43]}$. It has great advantages for dealing with complex nonlinear relationships, especially in experiments with small sample sizes. Using Matlab7.11.0 to program and optimize related parameters, in contrast to the ANN modeling method, the SVM separately establishes a nonlinear correlation analysis model of the relation between each influx component and the four model pharmacodynamic indexes, and then calculates the contribution of each variable to the total efficacy.In order to comprehensively assess the correlation between each component and efficacy, the values of each index were normalized according to the Mapminmax function.The related equation 6 is as follows: 


$$
x(i)=\frac{x_{0}(i)-\operatorname{Min}\left(x_{0}\right)}{\operatorname{Max}\left(x_{0}\right)-\operatorname{Min}\left(x_{0}\right)}
$$

Where $x_{0}(i)$ is the original plasma concentration or cell viability of each group,

Max/Min represents the maximum / minimum value of the plasma concentration or cell viability of each group.

Using LIBSVM software, selected RBF kernel function for SVM effect on the output variable. the final manuscript.

\section{Abbreviations}

$$
\text { NMT NaoMaiTong }
$$
calculation.Using the weight coefficient $w$ to judge the importance of input variables to the output variables. The expression is:

$$
w=\text { model.SVs }{ }^{\prime} * \text { model.sv_coef }
$$

If $w$ is greater than 0 , it indicates that the variable has a positive effect on the output variable; if it is smaller than 0 , it indicates that the variable has a negative effect on the output variable; if it is equal to 0 , it indicates that the variable has no

Author Contributions: Conceived and designed the experiments: S.-m.W. and L.-L.; Performed the experiments: L.-L. and Y.-Z.; Analyzed the data: Y.-Z., L.-L., X.-1.F.; Analyzed the data: L.-L., Y.-Z.; Wrote the paper: Y.-Z. All authors read and approved

Funding: This research received no external funding.

Acknowledgments: This study was financially supported by the National Natural Science Foundation of China (No. 81274060 and 81473413).

Conflict of interest: The authors declare that they have no conflict of interest. 
542

543 SVM Support Vector Machine

544 PK-PD Pharmacokinetic-pharmacodynamic

545 TCM Traditional Chinese Medicine

546 CCM Compound Chinese Medicine

547 DMSO Dimethyl Sulfoxide
549

550

551

552

553

554

555

556

557

558

559

560

561

562

563

564

565

\section{References}

[1] Hu, X.; De Silva, T.M.; Chen, J.; Faraci, F.M. Cerebral vascular disease and neurovascular injury in ischemic stroke. J.Circulation Research. 2017, 120, 449-471.

[2] Flynn, R.W.; Macwalter, R.S.; Doney, A.S. The cost of cerebral ischaemia. $J$. Neuropharmacology. 2008, 55, 250-6.

[3] Ildan Faruk; Göçer Alp I.; Tuna Metin; Polat Sait; Kaya Mehmet; Isbir Turgay; Çetinalp Erdal. The effects of the pre-treatment of intravenous nimodipine on $\mathrm{na}(+)-\mathrm{k}+/ \mathrm{mg}+2$ atpase, $\mathrm{ca}+2 / \mathrm{mg}+2$ atpase, lipid peroxidation and early ultrastructural findings following middle cerebral artery occlusion in the rat. J. Neurological Research. 2001, 23, 96-104.

[4] Turley, K.R.; Toledopereyra, L.H.; Kothari, R.U. Molecular mechanisms in the pathogenesis and treatment of acute ischemic stroke. J. Journal of Investigative Surgery. 2005, 18, 207-218.

[5] Shi, Y.; Jiang, X.; Zhang, L.; Pu, H.; Hu, X.; Zhang, W.; Cai, W.; Gao, Y.; Leak, R.K.; Keep, R.F.; Bennett, M.V.; Chen, J. Endothelium-targeted overexpression of heat shock protein 27 ameliorates blood-brain barrier disruption after ischemic brain injury. J. Proc Natl Acad Sci USA. 2017, 114, E1243. 
566

567

[6] Wu, Y.; Shang, Y.; Sun, S.G.; Liu, R.G.; Yang, W.Q. Protective effect of erythropoi-etin against 1-methyl-4-phenylpyridinium-induced neurodegenaration in PC12 cells. J. Neurosci Bull. 2007, 23, 156-164.

[7] Westerink, R.H.; Ewing, A.G. The PC12 cell as model for neurosecretion. J. Acta Physiologica. 2008, 192, 273-285.

[8] Hardy, J.; Selkoe, D.J. The amyloid hypothesis of Alzheimer's disease:progress and problems on the road to therapeutics. J. Science. 2002, 297, 353-356.

[9] Ma, W.; Yuan, L.; Yu, H.; Ding, B.; Xi, Y.; Feng, J.; Xiao, R. Genistein as a neuroprotective antioxidant attenuates redox imbalance induced by beta-amyloid peptides 25-35 in PC12 cells. J. Int J Dev Neurosci. 2010, 28, 289-295.

[10] Durukan, A.; Tatlisumak, T. Acute ischemic stroke: Overview of major experimental rodent models, pathophysiology, and therapy of focal cerebral ischemia. J. Pharmacology, Biochemistry and Behavior. 2007, 87, 179-197.

[11] Liu, P.K.; Grossman, R.G.; Hsu, C.Y.; Robertson, C.S. Ischemic injury and faulty gene transcripts in the brain. J. Trends in Neurosci. 2001, 24, 581-588.

[12] Chen, Y.H.; Du, G.H.;Zhang, J.T. Salvianolic acid B protects brain gainst injuries caused by ischemia-reperfusion in rats. J. Actaharmacol Sin. 2000, 21, 463-466.

[13] Sun, K.; Fan, J.; Han, J. Ameliorating effects of traditional Chinese medicine preparation, Chinese materia medica and active compounds on ischemia/reperfusion-induced cerebral microcirculatory disturbances and neuron damage. J. Acta Pharmaceutica Sinica B. 2015, 5, 8-24.

[14] Li, J.S.; Guo, M.D. Evaluation of clinical curative effect of NMT prescription on 
588

589

590

591

592

593

594

595

596

597

598

599

600

601

602

603

604

605

606

607

608

609

acute cerebral infarction. J. Chin. Med. 2005, 20, 563-565.

[15] Wu, C.; Lu, Z.; Rong, Y.; Zhu, G.; Liang, S.; Wang, S. The pharmacokinetic screening of multiple components of the nao mai tong formula in rat plasma by liquid chromatography tandem mass spectrometry combined with pattern recognition method and its application to comparative pharmacokinetics. J. Journal of Pharmaceutical \& Biomedical Analysis. 2016, 131, 345-354.

[16] Ren, X.Q.; Li, J.S.; Feng, Y.M.; Lu, Y.Q. Neuro-protective effect of Naomaitong to brain damage after focal cerebral ischemia reperfusion(I/R) in the aged rats. $J$. China Journal of Chinese Materia Medica. 2004, 29, 66-70.

[17] Chen, X.; Yang, Y.X.; Wang, S.M.; Wang, Z.H.; Su, Z.B.;Li, J.S.; Liang, S.W. Study on effects of extract in Naomaitong Formula on cerebral ischemia-reperfusion model based on NMR metabolomics. J. Chinese Traditional and Herbal Drugs. 2012.,43, 97-102.

[18] Rong, Y.; Feng, S.; Wu, C.; Wang, S.; Liang, S.; Liu, D. LC-high resolution-MS/MS analysis of chemical compounds in rat plasma after oral administration of nao-mai-tong and its individual herbs. $J$. Biomedical Chromatography. 2016, 31.

[19] Zhu, D.N.; Chun-Wei, W. U.; Chen, C.;Liang, S. W.;Wang, S. M. Predicting the material basis of compound Naomaitong for ischemic stroke based on molecular docking simulation. J Chinese Medicinal Materials. 2017, 40, 673-679.

[20] Gao, J.F.; Zhou, Y.L.; Li, J.S. Effect of Naomaitong on nuclear factor kinases 
610

611

612

613

614

615

616

617

618

619

620

621

622

623

624

625

626

627

628

629

630

631

NF-kB and nitric oxide synthase in aged rats with focal cerebral ischemia reperfusion.

J. Journal of Beijing University of Traditional Chinese Medicine. 2007, 30, 181-184.

[21] Li, J.S.; Gao, J.F.; Zhou, Y.L.; Liu, K. Neuro-protective effect of Naomaitong to inflammatory cascade response after focal cerebral ischemia reperfusion in aged rat. $J$.

China Journal of Chinese Materia Medica. 2006, 31, 1804-1807.

[22] Sheiner, L.B.; Stanski, D.R.; Vozeh, S.; Miller, R.D.; Ham, J. Simultaneous modeling of pharmacokinetics and pharmacodynamics: application to d-tubocurarine. J. Clinical Pharmacology \& Therapeutics. 1979, 25, 358.

[23] Vapnik V. Pattern recognition using generalized portrait method. J Autom Remote Control. 1963, 24, 774-780.

[24] Noble W.S. What is a support vector machine? J Nature Biotechnology. 2006, 24,1565 .

[25] Czock, D.; Keller, D.F.; Rasche, F.M.; Häussler, U. Pharmacokinetics and pharmacodynamics of systemically administered glucocorticoids. J. Clinical Pharmacokinetics. 2005, 44, 61-98.

[26] Lovern, M.; Underwood, M.; Nichols, G.; Song, I. PK/PD modeling supports the dose-escalation decisionin VIKING. J. Journal of the International AIDS Society. 2010, 13, 182.

[27] Duan, K.; Yuan, Z.; Guo, W.; Meng, Y.; Cui, Y.; Kong, D.; Zhang, L.; Wang, N. LC-MS/MS determination and pharmacokinetic study of five flavone components after solvent extraction/acid hydrolysis in rat plasma after oral administration of Verbena officinalis L. extract. J. J Ethnopharmacol. 2011, 135, 201-208. 
632

633

634

635

636

637

638

639

640

641

642

643

644

645

646

647

648

649

650

651

652

653

[28] Wang, L.; Wang, Z.; Wo S.; Lau C.B.; Chen, X.; Huang, M.; Lee V.H.; Chow

M.S.; Zuo, Z. A bio-activity guided in vitro pharmacokinetic method to improve the quality control of Chinese medicines, application to Si Wu Tang. J. J Ph Int arm. 2011, 406, 99-105.

[29] Zhu, H.; Bi, K.; Han, F.; Guan, J.; Zhang, X.; Mao, X.; Zhao, L.; Li, Q.; Hou, X.; Yin, R. Identification of the absorbed components and metabolites of Zhi-Zi-Da-Huang decoction in rat plasma by ultra-high performance liquid chromatography coupled with quadrupole-time-of-flight mass spectrometry. $J$. Journal of Pharmaceutical and Biomedical Analysis. 2015, 111, 277-287.

[30] Liu, X.; Wang, X.L.; Wu, L.; Li, H.; Qin, K.M.; Cai, H.; Pei, K.; Liu, T.; Cai, B.C. Investigation on the spectrum-effect relationships of Da-Huang-Fu-Zi-Tang in rats by UHPLC-ESI-Q-TOF-MS method. J. Journal of Ethnopharmacology. 2014, 154, 606-612.

[31] Shia, C.S.; Juang, S.H.; Tsai, S.Y.; Chang, P.H.; Kuo, S.C.; Hou, Y.C.; Chao, P.D. Metabolism and pharmaco- kinetics of anthraquinones in Rheum palmatum in Rats and ex vivo antioxidant activity. J. Planta Med. 2009, 75, 1386-1392.

[32] Jiang, P.; Liu, R.; Dou, S.; Liu, L.; Zhang, W.; Chen, Z.; Xu, R.; Ding, J. Analysis of the constituents in rat plasma after oral administration of Shexiang Baoxin pill by HPLC-ESI-MS/MS. J. Biomedical Chromatography. 2009, 23, 1333-1343.

[33] Wang, X.; Zhao, T.; Gao, X.; Dan, M.; Zhou, M.; Jia, W. Simultaneous determination of 17 ginsenosides in rat urine by ultra performance liquid chromatography-mass spectro- metry with solid-phase extraction. J. Analytica 
654

655

656

657

658

659

660

661

662

663

664

665

666

667

668

669

670

671

672

673

674

675

Chimica Acta. 2007, 594, 265-273.

[34] Miao, W.J.; Wang, Q.; Bo, T.; Ye, M.; Qiao, X.; Yang, W.Z.; Xiang, C.; Guan, X.Y.; Guo, D.A. Rapid characterization of chemical constituents and rats metabolites of the traditional Chinese patent medicine Gegen- Qinlian-Wan by UHPLC/DAD/qTOF-MS. J. Journal of Pharmaceutical and Biome-dical Analysis. 2013, 72, 99-108.

[35] Zhou, Q.L.; Wang, Y.F.; Yang, D.H.; Xu, F.; Zhao, X.; Zhang, L.; Liang, J.; Yang, X.W. Identification of the absorptive constituents and their metabolites in vivo of Puerariae Lobatae Radix decoction orally administered in WZS-miniature pigs by HPLC-ESI-Q-TOFMS. J. Biomedical Chromatography. 2013, 27, 1208-1218.

[36] Zuo, A.; Wang, L.; Xiao, H.; Li, L.; Liu, Y.; Yi, J. Identification of the absorbed components and metabolites in rat plasma after oral administration of Rhizoma Chuanxiong decoction by HPLC-ESI-MS/MS. J. Journal of Pharmaceutical and Biomedical Analysis. 2011, 56, 1046-1056.

[37] Xiong, Y.K.; Lin, X.; Liang, S.; Hong, Y.L.; Shen, L.; Feng, Y. Identification of senkyunolide I metabolites in rats using ultra performance liquid chromatography/quadrupole-time-of-flight tandem mass spectrometry. J. Journal of Pharmaceutical and Biomedical Analysis. 2013, 81-82, 178-186.

[38] Longa, E.Z.; Weinstein, P.R.; Carlson, S.; Cummins, R. Reversible middle cerebral artery occlusion without craniectomy in rats. J. Stroke. 1989, 20, 84-91.

[39] Cao, G.S.; Li, S.X.; Wang, Y.; Xu, Y.Q.; Lv, Y.N.; Kou, J.P.; Yu, B.Y. A combination of four effective components derived from Sheng-mai san attenuates 
676 hydrogen peroxide-induced injury in PC12 cells through inhibiting Akt and MAPK

677 signaling pathways. J. Chinese Journal of Natural Medicines. 2016, 14, 508-517.

678 [40] Fan, X.; Rong, Y.; Wang, S. Analysis of NaoMaiTong Metabolites Using

679 High-Performance Liquid Chromatography/High-Resolution Mass Spectrometry in 680 Rat Urine. J. Chromatographia. 2017, 4, 1-29.

681 [41] Mosmann, T. Rapid colorimetric assay for cellular growth and survival: 682 application to proliferation and cytotoxicity assays. J. Immunol. Methods. 1983, 65, $683 \quad 55-63$.

684 [42] Yang, X.; Wang, Y.; Luo, J.; Liu, S.; Yang, Z. Protective effects of YC-1 against 685 glutamate induced $\mathrm{PC} 12$ cell apoptosis. J. Cellular \& Molecular 686 Neurobiology. 2011, 31, 303-311.

687 [43] Vapnik V.N. An overview of statistical learning theory. J IE-EE Trans Neural 688 Netw. 1999, 10, 988-999.

689

690 